\title{
PROPERTIES OF SUBGROUPS OF SOLVABLE GROUPS THAT IMPLY THEY ARE NORMALLY EMBEDDED
}

\author{
ARNOLD FELDMAN \\ Franklin and Marshall College, Lancaster, PA 17604-3003, USA \\ e-mail: arny.feldman@fandm.edu
}

(Received 29 May, 2001; accepted 18 September, 2001)

\begin{abstract}
Recently, Ballester-Bolinches [1 and 2], Pedraza-Aguilera [2] and PerezRamos [2] have studied circumstances under which certain injectors and projectors, which are always pronormal, must be normally embedded. In this note we give a scheme for describing a minimal counterexample to a conjecture of the form: a subnormally embedded subgroup with properties $\alpha_{1}, \alpha_{2}, \ldots, \alpha_{n}$ is normally embedded, where $\alpha_{1}$, $\alpha_{2}, \ldots, \alpha_{n}$ satisfy certain conditions. We then show contradictions in certain cases involving finite solvable groups.
\end{abstract}

2000 Mathematics Subject Classification. 20D10.

The concept of normal embedding of a subgroup is powerful and interesting. Any normally embedded subgroup of a finite solvable group is an injector [3, VIII(3.8)], and there are a variety of known results showing that two weaker properties combined imply normal embedding in a finite solvable group. For example a subgroup is normally embedded if it is subnormally embedded and locally pronormal [3, p. 261]; if it is locally pronormal and a CAP subgroup [3, I(7.13)]; and if it is locally pronormal and system permutable [3, I(7.13)]. Recently, Ballester-Bolinches [1, 2], Pedraza-Aguilera [2], and Perez-Ramos [2] have studied circumstances under which certain injectors and projectors, which are always pronormal, must be normally embedded. In this note we give a scheme for describing a minimal counterexample to a conjecture of the form: a subnormally embedded subgroup with properties $\alpha_{1}, \alpha_{2}, \ldots, \alpha_{n}$ is normally embedded, where $\alpha_{1}, \alpha_{2}, \ldots, \alpha_{n}$ satisfy certain conditions. We then show contradictions in certain cases, proving:

COROLlary 1. If $G$ is a finite solvable group, and $U$ is a subgroup of $G$ that is $S$-quasinormally embedded and pronormal in $G$, then $U$ is normally embedded in $G$.

This implies:

COROLlary 3. If $G$ is a finite solvable group, and $U$ is a subgroup of $G$ that is permutably embedded and pronormal in $G$, then $U$ is normally embedded in $G$.

Each of these two results generalizes a result found in [1] or [2], as described below. We also prove:

THEOREM 3. If $G$ is a finite solvable group, and $U$ is a subgroup of $G$ that is subnormally embedded and is Frattini on intersections in $G$, then $U$ is normally embedded in $G$. 
The reason that these results appear as corollaries rather than theorems is that the methods we apply generalize from analysis of Sylow subgroups to analysis of $\mathbf{H}$-projectors, where $\mathbf{H}$ is a solvable Schunck class. We discuss some of the implications of this fact below.

All groups in this paper are finite and solvable, and the notation used is that of Doerk and Hawkes' book Finite Soluble Groups [3]. Here are some definitions required below. In these definitions, $r$ is a prime, and $\mathbf{H}$ is a Schunck class of solvable groups, so that any solvable group has a unique conjugacy class of H-projectors by [3, III(3.21)].

A subgroup $A$ is permutable in $G$ if for every subgroup $B$ of $G, A B$ is a subgroup of $G$.

A subgroup $A$ is $S$-quasinormal in $G$ if, for every Sylow subgroup $B$ of $G, A B$ is a subgroup of $G$. Hence a subgroup is $\mathrm{S}$-quasinormal if and only if it permutes with every Hall subgroup of $G$. Also, by $\left[3, \mathrm{I}(4.21)(\mathrm{a})\left(\mathrm{b}^{\prime}\right)\right]$, an S-quasinormal subgroup is subnormal.

A subgroup $A$ is pronormal in $G$ if for each $g \in G, A$ is conjugate to $A^{g}$ by an element of $\left\langle A, A^{g}\right\rangle$.

A subgroup $A$ is abnormal in $G$ if for each $g \in G, g \in\left\langle A, A^{g}\right\rangle$.

By $[3, \mathrm{I}(6.21)(\mathrm{a})]$, the normalizer of a pronormal subgroup is abnormal. This implies that $A$ is abnormal in $G$ if and only if $A$ is both pronormal and self-normalizing in $G$.

A subgroup $A$ of $G$ satisfies the Frattini argument in $G$ if for every normal subgroup $L$ of $G$ with $A \leq L, G=L N_{G}(A)$. This is equivalent to the property that for each normal subgroup $L$ of $G$, all conjugates of $A$ in $G$ are conjugate to $A$ in $L$.

A subgroup $A$ is Frattini on intersections in $G$ if, whenever $K$ is subnormal in $G$, $A \cap K$ satisfies the Frattini argument in $K$.

A subgroup $A$ is pronormalizing in $G$ if for every subnormal subgroup $L$ of $G$ that contains $A, N_{L}(A)$ is abnormal in $L$. By [3, I(6.3)(a) and $\left.\mathrm{I}(6.21)(\mathrm{a})\right]$, if $A$ is pronormal in $G$ then $A$ is pronormalizing in $G$.

The subnormal closure $\left\langle A^{. G}\right\rangle$ of $A$ in $G$ is the unique smallest subnormal subgroup of $G$ containing $A$.

The normal closure $A^{G}$ of $A$ in $G$ is the unique smallest normal subgroup of $G$ containing $A$.

We will use a (possibly subscripted) $\alpha$ to denote a subgroup property, as follows: $U \alpha G$ will indicate that $U$ is a subgroup of $G$ having some property, for example, $U$ is subnormal in $G$. We say a subgroup $A$ is $\mathbf{H}$ - $\alpha$-ly embedded in $G$ if each $\mathbf{H}$-projector of $A$ is an H-projector of a subgroup $X$ of $G$ such that $X \alpha G$. For example, a subgroup $A$ is $\mathbf{H}$-normally embedded in $G$ if each $\mathbf{H}$-projector of $A$ is an $\mathbf{H}$-projector of a normal subgroup of $G$.

Note that if $\mathbf{H}=\mathbf{R}$, the Schunck class of $r$-groups for some prime $r$, then the $\mathbf{R}$-projectors are the Sylow $r$-subgroups, so R-normally embedded means $r$-normally embedded as in [3, I(7.1)(a)], so a subgroup is normally embedded [3, I(7.1)(b)] if and only if it is R-normally embedded for each prime $r$. Similarly, a subgroup is subnormally embedded [3, p. 261] if and only if it is $\mathbf{R}$-subnormally embedded for each prime $r$, S-quasinormally embedded [2, p. 244] if and only if it is R-S-quasinormally embedded for each prime $r$, and permutably embedded [1, p. 1] if and only if it is $\mathbf{R}$-permutably embedded for each prime $r$.

The theorems below are stated and proved in terms of these generalized embedding properties, and the corollaries apply to the more standard embedding properties. Thus the reader who is interested in the corollaries but not the more general theorems is 
encouraged to substitute Sylow $r$-subgroup for $\mathbf{H}$-projector in the proofs below, and ignore the few statements that become tautologies.

We need one easy lemma for our induction arguments.

Lemma 1. If $X \alpha G$ implies $X N / N \alpha G / N$ for $N$ normal in $G$, then if $U$ is $\mathbf{H}-\alpha$-ly embedded in $G, U N / N$ is $\mathbf{H}-\alpha$-ly embedded in $G / N$.

Proof. Suppose $B / N$ is an $\mathbf{H}$-projector of $U N / N$, where $U$ is $\mathbf{H}-\alpha$-ly embedded in $G$. Let $C$ be an $\mathbf{H}$-projector of $U$, so by [3, $\operatorname{III}(3.3)], C N / N$ is an $\mathbf{H}$-projector of $U N / N$, and by [3, III(3.21)], $B / N=(C N / N)^{x N}$, where $x \in U$. Let $A=C^{x}$, so $A$ is an $\mathbf{H}$-projector of $U$ and $B=A N$. Thus $A$ is also an H-projector of $X$, where $X \alpha G$. Then $B / N$ is an $\mathbf{H}$-projector of $X N / N$ by [3, $\operatorname{III}(3.3)]$, and $X N / N \alpha G / N$ by assumption. Hence $U N / N$ is $\mathbf{H}-\alpha$-ly embedded in $G / N$, as claimed.

Now we prove the following theorem.

THEOREM 1. Suppose that $\alpha_{1} \ldots \alpha_{n}$ are properties such that for any group $G$, if $K$ is normal in $G$, then for $i=1 \ldots n, U \alpha_{i} G$ implies $U K / K \alpha_{i} G / K$. Let $G$ be a finite solvable group of minimal order having a subgroup $U$ with $\mathbf{H}$-projector A such that $U$ is $\mathbf{H}$-subnormally embedded in $G$ and $U \alpha_{i} G$ for $i=1 \ldots n$, but $U$ is not $\mathbf{H}$-normally embedded in $G$. Then $G$ satisfies the following conditions:

(a) $\operatorname{core}_{G}(A)=1$;

(b) there exists a minimal normal subgroup $N$ of $G$, such that $A^{G}=A N$ and $A^{G}$ is an elementary abelian p-group, which implies $A$ is subnormal in $G$;

(c) $N_{G}(U) \leq N_{G}(A)$;

(d) $A \in \operatorname{Syl}_{p}(U)$.

Proof. Suppose $G$ and $U$ satisfy the hypotheses, and let $N$ be a minimal normal subgroup of $G$, so $N$ is an elementary abelian $p$-group for some prime $p$. Then $A N / N$ is an $\mathbf{H}$-projector of $U N / N$ by [3, III(3.3)].

By Lemma 1, $U N / N$ is $\mathbf{H}$-subnormally embedded in $G / N$, and $U N / N \alpha_{1} G / N$, $U N / N \alpha_{2} G / N, \ldots U N / N \alpha_{n} G / N$ by assumption, so by minimality of $G, U N / N$ is $\mathbf{H}$ normally embedded in $G / N$. Hence $A N / N$ is an H-projector of some normal subgroup $J / N$ of $G / N$. Now if $\operatorname{core}_{G}(A)>1$, A contains such an $N$, so $A / N$ is an $\mathbf{H}$-projector of $J / N$, so by [3, III(3.7)], $A$ is an H-projector of the normal subgroup $J$ of $G$, a contradiction establishing (a).

Now let $K=\left\langle A^{*}{ }^{G}\right\rangle$, so $A$ is an $\mathbf{H}$-projector of $K$, because $K$ is contained in any subnormal subgroup of $G$ that contains $A$, and $A$ is an $\mathbf{H}$-projector of one of these because $U$ is $\mathbf{H}$-subnormally embedded in $G$. Now $A$ is not an $\mathbf{H}$-projector of $A^{G}$ because $U$ is not $\mathbf{H}$-normally embedded in $G$, so $K<A^{G}$. Let $N$ be a minimal normal subgroup of $G$ contained in $A^{G}$. Then $A N / N$, being an $\mathbf{H}$-projector of some normal subgroup $J / N$ of $G / N$, is pronormal in $G / N$. For if $x \in G / N,(A N / N)^{x}$ is an $\mathbf{H}$ projector of $(J / N)^{x}=J / N$, so $A N / N$ and $(A N / N)^{x}$ are both $\mathbf{H}$-projectors of their join $\left\langle A N / N,(A N / N)^{x}\right\rangle \leq J / N$; hence they are conjugate in that join.

Thus $\left\langle(A N / N)^{\cdot . G / N}\right\rangle=(A N / N)^{(G / N)}$ [3, p. 261]. Now $(A N / N)^{(G / N)}=\left((A N)^{G}\right) /$ $N=A^{G} / N$, so $\left\langle(A N / N)^{. . G / N}\right\rangle=A^{G} / N$. But $K N / N$ is subnormal in $G / N$ and contains $A N / N$, so $A^{G} / N=\left\langle(A N / N)^{. . G / N}\right\rangle \leq K N / N \leq A^{G} / N$; hence $K N=A^{G}$. Therefore, $K N$ is normal in $G$.

Then $K$ is core free, for if $K$ contains any minimal normal subgroup of $G$ we may choose that subgroup to be $N$, implying $K=K N=A^{G}$, a contradiction. Now $K$, being subnormal in $G$, is normalized by every minimal normal subgroup of $G$ 
by [3, A(14.3)], so $K$ is normal in $K N$. Thus $K$ contains $O^{p}\left(A^{G}\right)$, because $K N / K$ is a $p$-group, so $O^{p}\left(A^{G}\right)=1$ and $A^{G}$ is a $p$-group. And by [3, $\left.\mathrm{A}(9.6)\right], K$ contains $A^{G}$, s Frattini subgroup, which is normal in $G$ and therefore trivial because $K$ is core free, so $A^{G}$ is elementary abelian. Thus $A$ is normal in $A^{G}$, and therefore subnormal in $G$, so $K=A$ and $A^{G}=A N$. This establishes (b).

Now $A$ is pronormal in $U$ because it is an $\mathbf{H}$-projector of $U$, and $A$ is subnormal in $U$ because it is subnormal in $G$, so $A$ is normal in $U$ by [3, $\mathrm{I}(6.3)(\mathrm{d})]$. Thus $A$ is the unique $\mathbf{H}$-projector of $U$. Now suppose $x \in N_{G}(U)$. Then $A^{x}$ is an $\mathbf{H}$-projector of $U^{x}=U$, so $A^{x}=A$. Hence $N_{G}(U) \leq N_{G}(A)$, establishing (c).

Let $\pi$ be the characteristic of the Schunck class $\mathbf{H}[\mathbf{3}, \mathrm{II}(1.2)(\mathrm{b})]$. Because $A$ is a $p$-group, $A$ has a homomorphic image of order $p$, which is in $\mathbf{H}$ because $A$ is by [3, III(2.7)]. Thus $p \in \pi$. Then $U / A=N_{U}(A) / A$ is a $\pi^{\prime}$-group by [3, III(4.8)], so $A \in \operatorname{Syl}_{p}(U)$, completing the proof of (d).

We are now in position to prove:

THEOREM 2. If $G$ is a finite solvable group and $\mathbf{H}$ is a Schunck class, and $U$ is a subgroup of $G$ that is $\mathbf{H}$-S-quasinormally embedded and pronormalizing in $G$, then $U$ is $\mathbf{H}$-normally embedded in $G$.

This immediately yields:

COROLLARY 1. If $G$ is a finite solvable group, and $U$ is a subgroup of $G$ that is $S$ quasinormally embedded and pronormalizing in $G$, then $U$ is normally embedded in $G$. Thus if $U$ is $S$-quasinormally embedded and pronormal in $G$, then $U$ is normally embedded in $G$.

Ballester-Bolinches, Pedraza-Aguilera, and Perez-Ramos have given examples of subnormally embedded pronormal subgroups in finite solvable groups that are not normally embedded [2, Theorem 4]. Thus if every pronormal subgroup with properties $\alpha_{1}, \ldots, \alpha_{n}$ is normally embedded, together properties $\alpha_{1}, \ldots, \alpha_{n}$ must be stronger, at least when combined with pronormality, than subnormal embedding. Corollary 1 shows that S-quasinormally embedded is a sufficiently strong property.

Proof of Theorem 2. If $U$ is pronormalizing in $G$ and $K$ is normal in $G$, it is easy to see that $U K / K$ is pronormalizing in $G / K$ : If $U K / K \leq L / K$ and $L / K$ is subnormal in $G / K$, then $U \leq L$ and $L$ is subnormal in $G$, so if $x K \in L / K$, so $x \in L$, we have $x \in\left\langle N_{L}(U), N_{L}(U)^{x}\right\rangle$ because $N_{L}(U)$ is abnormal in $L$. Hence $x K \in\left\langle N_{L}(U), N_{L}(U)^{x}\right\rangle K / K \leq\left\langle N_{L / K}(U K / K), N_{L / K}(U K / K)^{x K}\right\rangle$, and $N_{L / K}(U K / K)$ is abnormal in $L / K$. Hence $U K / K$ is pronormalizing in $G / K$. And if $U$ is $\mathbf{H}$-Squasinormally embedded in $G$ and $K$ is normal in $G$, then $U K / K$ is H-S-quasinormally embedded in $G$ by Lemma 1, using the easily proved fact that if $X$ is S-quasinormal in $G$, then $X K / K$ is S-quasinormal in $G / K$.

Because an S-quasinormal subgroup is subnormal, an $\mathbf{H}$-S-quasinormally embedded subgroup is $\mathbf{H}$-subnormally embedded. Thus if we consider a minimal counterexample to Theorem 2, the hypotheses of Theorem 1 apply, with $n=2$ and properties $\alpha_{1}$ and $\alpha_{2}$ being pronormalizing and $\mathbf{H}$-S-quasinormally embedded. Hence we have the conclusions of Theorem 1 , with $G, U$, and $A$ as contained therein.

Thus $A$ is an $\mathbf{H}$-projector for some $\mathrm{S}$-quasinormal subgroup $X$ of $G$, and $A$ is subnormal in $G$ by Theorem 1(b). Then $A \in \operatorname{Syl}_{p}(X)$ by the same argument used in the proof of Theorem 1(d) to show that $A \in \operatorname{Syl}_{p}(U)$. Let $W$ be any Hall $p^{\prime}$-subgroup of $G$. Then $X W$ is a subgroup of $G$ because $X$ is S-quasinormal, and $A \in \operatorname{Syl}_{p}(X W)$. But 
$A$ is subnormal in $G$, so it is subnormal in $X W$, and $A$ is pronormal in $X W$, so it is normal in $X W$.

Thus $A$ is normalized by every Hall $p^{\prime}$-subgroup of $G$, so $A$ is normalized by $O^{p}(G)$, the join of all Hall $p^{\prime}$-subgroups of $G$. But $A$ is normal in $A^{G}$, so the normal subgroup $A^{G} O^{p}(G)$ of $G$ is contained in $N_{G}(A)$. Because $A$ is not normal in $G, A^{G} O^{p}(G)<G$. Now any Hall $p^{\prime}$-subgroup of $U$, say $V$, is contained in $O^{p}(G)$, so $U=A V$ is contained in $A^{G} O^{p}(G)$. Similarly, $X$ is contained in $A^{G} O^{p}(G)$. But $G / A^{G} O^{p}(G)$ is a $p$-group, so there exists some normal subgroup. $L$ of index $p$ in $G$ that contains $A^{G} O^{p}(G)$ and therefore $U$ and $X$.

Because $U$ is pronormalizing in $G, U$ is pronormalizing in $L$ by transitivity of subnormality. Because $X$ is S-quasinormal in $G$, and contained in $L$, it is easy to see that $X$ is $\mathrm{S}$-quasinormal in $L$ : Suppose $Q$ is a Sylow $q$-subgroup of $L$. Then $Q \leq Q_{0}$, a Sylow $q$-subgroup of $G$. Then $X Q_{0}$ is a subgroup of $G$, so $X Q_{0} \cap L=X\left(Q_{0} \cap L\right)=X Q$ is a subgroup of $L$, and $X$ is $\mathrm{S}$-quasinormal in $L$. (Note that $X \leq L$ was sufficient we did not need the normality of $L$ in $G$.) Thus $U$ is $\mathbf{H}$-S-quasinormally embedded in $L$. Hence by minimality of $G, U$ is $\mathbf{H}$-normally embedded in $L$. Hence $A$ is an $\mathbf{H}$ projector of some normal subgroup $Y$ of $L$. By the argument used in Theorem 1(b) $A$ is pronormal in $L$, so $A$ is normal in $L$. Hence $N_{G}(A)=L$. But $U$ is pronormalizing in $G$, so $N_{G}(U)$ is abnormal and therefore self-normalizing in $G$ by [3, I(6.21) (a) and (b)], and so is any subgroup that contains it [3, I(6.20) (b) and (c)]. But $L=N_{G}(A)$ contains $N_{G}(U)$ by Theorem 1(c). Thus $L$ is a proper subgroup of $G$ that is self-normalizing and normal in $G$. This contradiction establishes the theorem.

Because $\mathbf{H}$-projectors are pronormal, we immediately obtain the following.

Corollary 2 [2, Theorem 2]. If $\mathbf{H}$ is a Schunck class and $U$ is an $S$-quasinormally embedded $\mathbf{H}$-projector of a finite solvable group $G$, then $U$ is normally embedded in $G$.

It is clear from the definitions that a permutable subgroup is S-quasinormal, so a permutably embedded subgroup is S-quasinormally embedded. Thus we immediately have:

COROLlary 3. If $G$ is a finite solvable group, and $U$ is a subgroup of $G$ that is permutably embedded and pronormal in $G$, then $U$ is normally embedded in $G$.

Because $F$-injectors are pronormal, we immediately obtain the following.

COROLlary 4. If $F$ is a Fitting set and $U$ is a permutably embedded F-injector of a finite solvable group $G$, then $U$ is normally embedded in $G$.

This result slightly generalizes [1, Theorem 6], which gives the same conclusion, but is restricted to Fitting classes.

Now we present an example, due to T.O. Hawkes, of a solvable group $G$ with a pronormalizing subgroup $U$ that is not pronormal in $G$.

Let $S$ be $S L(2,3)$, which is easily seen to act irreducibly and faithfully on an elementary abelian group $N$ of order 9 . Let $G=N S$, the semidirect product of $N$ with $S$ induced by this action. Then $N$ is the unique minimal normal subgroup of $G$, and $S$ contains a subgroup $Q$ that is a quaternion group of order 8 , is normal in $S$, and is a Sylow 2-subgroup of $G$. Let $U$ be a Sylow 3-subgroup of $S$, so $N U$ is a Sylow 3subgroup of $G$. Also, $[Q, U]=Q$, and $C_{Q}(U)=Z(Q)$. Thus $U^{Q}$ contains $[Q, U] U=S$, and, being nontrivial, $U^{G}$ contains $N$, so $U^{G}=G$. Thus if $U \leq L$ and $L$ is subnormal in $G$, then $L=G$. It is not difficult to show that $Z(Q) U$ is self-normalizing in $G$, and it is 
clearly nilpotent, so it is a Carter subgroup of $G$. Hence $N_{G}(U)$ contains the abnormal subgroup $Z(Q) U$, so $N_{G}(U)$ is abnormal in $G$. Hence $U$ is pronormalizing in $G$.

But $U$ is not pronormal in $G$. For if $U$ is pronormal in $G$, it is also pronormal in the 3-group $N U$, in which it is subnormal, so $U$ is normal in $N U$. But then $[N, U] \leq N \cap U=1$, and $U$ centralizes $N$, a contradiction. Thus a pronormalizing subgroup of a solvable group may fail to be pronormal in that group.

Because of the example in [2, Theorem 4], if every subnormally embedded subgroup with properties $\alpha_{1}, \ldots, \alpha_{n}$ is normally embedded, together properties $\alpha_{1}, \ldots, \alpha_{n}$ must be stronger, at least when combined with subnormally embedded, than pronormality. Now every pronormal subgroup satisfies the Frattini argument $[3, I(6.3)(b)]$, so there are subnormally embedded subgroups that satisfy the Frattini argument that are not normally embedded. On the other hand, in [4] we have proved that if $F$ is a Fitting set, then a subnormally embedded $F$-injector is normally embedded.

Note that if $F$ is a Fitting set of $G$, every $F$-injector $V$ of $G$ is pronormal in $G$ [3, VIII(2.14)(a)], and if $K$ is subnormal in $G, V \cap K$ is an $F$-injector of $K$ by [3, VIII(2.6)]. Note that this means that an $F$-injector of $G$ is Frattini on intersections in $G$. Here we strengthen the result from [4] somewhat.

THEOREM 3. If $G$ is a finite solvable group, and $U$ is a subgroup of $G$ that is subnormally embedded and is Frattini on intersections in $G$, then $U$ is normally embedded in $G$.

We begin with a pair of lemmas.

Lemma 2. (i) If $A$ is Frattini on intersections in $G$ and $K$ is subnormal in $G$, then $A \cap K$ is Frattini on intersections in $K$.

(ii) If $A$ is Frattini on intersections in $G$ and $N$ is normal in $G$, then $A N / N$ is Frattini on intersections in $G / N$.

Proof of (i). Suppose $A$ is Frattini on intersections in $G$ and $K$ is subnormal in $G$. We need to prove that $A \cap K$ is Frattini on intersections in $K$. Let $B=A \cap K$, and suppose $J$ is subnormal in $K$. We need only show that $B \cap J$ satisfies the Frattini argument in $J$. But $J$ is subnormal in $K$, which is subnormal in $G$, so $J$ is subnormal in $G$, and $B \cap J=A \cap K \cap J=A \cap J$, so to establish (i), we need only note that $A \cap J$ satisfies the Frattini argument in $J$ because $A$ is Frattini on intersections in $G$.

Proof of (ii). Now suppose $C$ is a subgroup that satisfies the Frattini argument in $G$, and $N$ is a normal subgroup of $G$. If $C N / N \leq L / N \leq G / N$, where $L / N$ is normal in $G$, then $C N \leq L$, which is normal in $G$. Now $G=N_{G}(C) L$, so $G=N_{G}(C N) L$, and $G / N=N_{G / N}(C N / N)(L / N)$, so $C N / N$ satisfies the Frattini argument in $G / N$. Now suppose $A$ is Frattini on intersections in $G$, and $N$ is normal in $G$. Consider $A N / N$. Suppose $K / N$ is subnormal in $G / N$. Now $A N / N \cap K / N=(A N \cap K) / N=(A \cap K) N / N$. But $A \cap K$ satisfies the Frattini argument in $K$, because $K$ is subnormal in $G$ and $A$ is Frattini on intersections in $G$, so with $A \cap K$ in place of $C$ and $K$ in place of $G$, the above argument implies that $(A \cap K) N / N$ satisfies the Frattini argument in $K / N$. Hence $A N / N$ is Frattini on intersections in $G / N$, establishing (ii).

LEMMA 3. Whenever $A$ is subnormally embedded in $G$ and $K$ is subnormal in $G$, then $A \cap K$ is subnormally embedded in $K$.

Proof. Suppose $r$ is a prime dividing $|A \cap K|$. Then $r$ divides $|A|$, so if $R$ is a Sylow $r$-subgroup of $A, R \in \operatorname{Syl}_{r}(X)$, where $X$ is subnormal in $G$. Now $X \cap K$ is 
subnormal in $X$ by [3, A(14.1) (a)], so $R \cap K \in \operatorname{Syl}_{r}(X \cap K)$ by repeated application of [3, A(6.4)(a)]. Similarly, $R \cap K \in \operatorname{Syl}_{r}(A \cap K)$. But note that $X \cap K$ is subnormal in $K$, so a Sylow $r$-subgroup of $A \cap K$ is a Sylow $r$-subgroup of a subnormal subgroup of $K$. Hence $A \cap K$ is subnormally embedded in $K$. (Note that $X \cap K$ is also subnormal in $G$, so $A \cap K$ is actually subnormally embedded in $G$, but we do not need that result here.)

Proof of Theorem 3. By Lemma 2(ii), we may apply Theorem 1, where $n=1$ and $U \alpha G$ means $U$ is Frattini on intersections in $G$. Hence a minimal counterexample to Theorem 3 satisfies the conclusions of Theorem 1. Consider a composition series of $G$ that contains $A^{G}$, and let $L$ be the (normal) maximal proper subgroup of $G$ in that series. Now $U \cap L$ is Frattini on intersections in $L$ by Lemma 2(i), and $U \cap L$ is subnormally embedded in $L$ by Lemma 3 . Hence by minimality of $G, U \cap L$ is normally embedded in $L$. Now $A \leq A^{G} \leq L$, so $A \in \operatorname{Syl}_{p}(U \cap L)$. Hence $A \in \operatorname{Syl}_{p}(Y)$, where $Y$ is normal in $L$. But $A$ is subnormal in $G$, so $A$ is subnormal and pronormal in $Y$, so it is normal and therefore characteristic in $Y$, and normal in $L$. Hence $L=N_{G}(A)$. But $U \leq N_{G}(U) \leq N_{G}(A)$ by Theorem $1(\mathrm{c})$, so $N_{G}(U) \leq L$ and $U \leq L$. But $U$ satisfies the Frattini argument in $G$, so $G=L N_{G}(U)$. Hence $G=L$. This contradiction establishes the theorem.

AN EXAMPLE. We now illustrate the significance of the assumptions of Theorems 2 and 3 with a group with subgroups satisfying the conclusions of Theorem 1, but not those of Theorems 2 and 3. We construct a group $G$ with subgroups $U_{1}$ and $U_{2}$, each of which is (3-)subnormally embedded but not (3-)normally embedded in $G$. The subgroup $U_{1}$ is neither pronormal nor pronormalizing, but it is 3-S-quasinormally embedded, i.e. H-S-quasinormally embedded, where $\mathbf{H}$ is the Schunk class of 3groups. On the other hand, $U_{2}$ is abnormal as well as pronormal, but it is neither 3-S-quasinormally embedded nor Frattini on intersections. Thus $G$ with $U_{1}$ illustrates the necessity of the assumption of pronormalizing in Theorem 2, while $G$ with $U_{2}$ reveals the necessity of the Frattini on intersections assumption of Theorem 3, as well as the necessity of the $\mathbf{H}$-S-quasinormally embedded assumption of Theorem 2.

Let $L$ be the extra-special group of order 27 with exponent 3. Then by [3, p. 79], $L=\left\langle x, y: x^{3}=1, y^{3}=1,\langle[x, y]\rangle=Z(L)\right\rangle$. Furthermore, by [3, $\left.\mathrm{A}(20.8)\right], L$ has an automorphism $z$ of order 2 such that $x^{z}=x^{-1}$ and $y^{z}=y$, so $[x, y]^{z}=[x, y]^{-1}$. Let $G$ be the semidirect product $L\langle z\rangle$ induced by the action of $\langle z\rangle$ on $L$. Set $A_{1}=\langle x\rangle$, $U_{1}=A_{1}\langle z\rangle, A_{2}=\langle y\rangle, U_{2}=A_{2}\langle z\rangle$, and $N=\langle[x, y]\rangle$.

It is not difficult to show that neither $A_{1}$ nor $A_{2}$ is normal in $G$, but each is subnormal in $G$. Thus each is (3-)subnormally embedded in $G$. Obviously $\langle z\rangle$ is (2-) subnormally embedded in $G$, because it is a Sylow 2-subgroup of $G$. Hence $U_{1}$ and $U_{2}$ are each subnormally embedded in $G$.

It is clear that $N$ is a minimal normal subgroup of $G$, and that $A_{1} N$ and $A_{2} N$ are each normal in $L$ and normalized by $z$, so $A_{1}^{G}=A_{1} N$ and $A_{2}^{G}=A_{2} N$. Hence neither $A_{i}$ is a Sylow 3-subgroup of $A_{i}^{G}$, so neither $A_{i}$ is (3-)normally embedded in $G$, and neither $U_{i}$ is normally embedded in $G$.

Let $M_{1}=A_{1} N\langle z\rangle=U_{1} N$; it is not hard to check that $M_{1}=N_{G}\left(A_{1}\right)$ and that $U_{1}$ is self-normalizing in $G$. Note that $A_{1} N$ is normal in $G$, and $z$ is centralized by $y$, so $M_{1}$ is normalized by $y$, and $M_{1}$ is normal in $G=M_{1} A_{2}$. Thus $N_{G}\left(U_{1}\right)$ is not abnormal in $G$ by $[3, \mathrm{I}(6.20)]$, so $U_{1}$ is not pronormal(izing) in $G$.

We can see that $G, U_{1}$, and $A_{1}$ satisfy the conclusions of Theorem 1 , where $\mathbf{H}$ is the Schunck class of 3-groups, so the $\mathbf{H}$-projectors are the Sylow 3-subgroups. In this 
case $U_{1}$ is $\mathbf{H}$-S-quasinormally embedded means that each Sylow 3-subgroup of $U_{1}$ is a Sylow 3-subgroup of a subgroup $X$ of $G$ that permutes with every Sylow subgroup of $G$. A check that $A_{1}$ is $\mathrm{S}$-quasinormal in $G$ is straightforward. Thus this example reveals the necessity of the pronormalizing condition in Theorem 2; the argument used in the proof of Theorem 2 breaks down because $A$ can in fact be normalized by every Sylow 2-subgroup of $G$, and therefore by $O^{3}(G)$. But here $O^{3}(G) \leq N_{G}\left(A_{1}\right)$, so $U_{1} \leq A_{1}^{G} O^{3}(G)=M_{1}$. The contradiction in Theorem 2 came because $N_{G}(U)$ was contained in no normal subgroup of $G$; here $N_{G}\left(U_{1}\right) \leq M_{1}$, and $M_{1}$ is normal in $G$. Note finally that we have an example here of an S-quasinormally embedded subgroup that has a Sylow 3-subgroup that is S-quasinormal (not just S-quasinormally embedded) and not (3-)normally embedded.

We omit the argument that $U_{2}$ is abnormal (and therefore pronormal(izing)) in $G$, but that $U_{2}$ is neither S-quasinormally embedded nor Frattini on intersections in $G$; it follows from the relevant definitions without complication.

It is worth noting that the same sort of construction, using the description of extraspecial groups and their automorphisms in [3, p. 79], allows the replacement in these examples of the prime 3 by any odd prime $p$, and the replacement of the prime 2 by any prime divisor of $p-1$.

Finally, it is not difficult to show that if $\mathbf{J}$ is defined to be the Schunck class $h\left(\left\{S_{3}\right\}\right)$ consisting of finite solvable groups none of whose epimorphic images is isomorphic to the symmetric group on 3 letters, then $G$ is a group minimal with respect to having subnormally embedded $\mathbf{J}$-projectors that are not normally embedded, and $U_{2}$ is a $\mathbf{J}$-projector of $G$. Thus $G$ is the kind of example described in [2, Theorem 4]. Because all nilpotent groups are members of $\mathbf{J}, \mathbf{J}$-projectors are always self-normalizing by [3, III(4.9)], so the abnormality of $U_{2}$ in $G$ is no surprise.

AcKnowledgements. The author wishes to thank Adolfo Ballester-Bolinches, Peter Hauck, Martin Newell, Trevor Hawkes, James Beidleman, and Rex Dark for valuable conversations, and the first three mentioned for conjecturing several of these results. The author also wishes to thank Franklin and Marshall College for its generous Sabbatical policy, NUI, Galway for the warm welcome and use of facilities, and the referee for helpful suggestions.

\section{REFERENCES}

1. A. Ballester-Bolinches, Permutably embedded subgroups of finite solvable groups, Arch. Math. 65 (1995), 1-7.

2. A. Ballester-Bolinches, M. C. Pedraza-Aguilera and M. D. Perez-Ramos, Characterizations of Schunck classes of finite soluble groups, J. Algebra 202 (1998), 243-249.

3. K. Doerk and T. Hawkes, Finite soluble groups (Walter de Gruyter, 1992).

4. A. Feldman, Conditions for which Fischer $F$-subgroups in finite solvable groups are F-injectors, Arch. Math. 73(6) (1999), 401-406.

5. The GAP Group, GAP - Groups, Algorithms, and Programming, Version 4.1; Aachen, St. Andrews, 1999. (http://www-gap.dcs.st-and.ac.uk/gap) 